\title{
Late and Reversible Kidney-Lung Failure after Intra-Bladder BCG Therapy
}

\author{
Olivier Mat ${ }^{{ }^{*}}$, Rim Kada ${ }^{1}$, Patrick Philippart ${ }^{2}$, Quentin Mat ${ }^{2}$, Steffy Larroze ${ }^{2}$, Myriam Remmelink ${ }^{3}$, \\ Selda Aydin ${ }^{4}$, Vincent Colombie ${ }^{5}$ \\ ${ }^{1}$ Department of Nephrology of EpiCURA, Ath, Belgium \\ ${ }^{2}$ Department of Surgery of EpiCURA, Ath, Belgium \\ ${ }^{3}$ Department of Lung Pathology, Hopital Erasme, Université Libre de Bruxelles, Brussels, Belgium \\ ${ }^{4}$ Department of Renal Pathology, Cliniques Universitaires St-Luc, Université Catholique de Louvain, Brussels, Belgium \\ ${ }^{5}$ Department of Infectious Diseases of EpiCURA, Ath, Belgium \\ Email: *Olivier.mat@epicura.be
}

Received May 9, 2013; revised June 15, 2013; accepted July 3, 2013

Copyright (C) 2013 Olivier Mat et al. This is an open access article distributed under the Creative Commons Attribution License, which permits unrestricted use, distribution, and reproduction in any medium, provided the original work is properly cited.

\begin{abstract}
We observed a 76-year-old man who presented "acute kidney-lung failure" 9 months after intravesical Bacillus Calmette-Guérin (BCG) adjuvant treatment for a T1 bladder cancer. He had inflammatory infiltration on chest radiography and required dialysis for acute renal failure. A percutaneous renal biopsy was performed and revealed tubulointerstitial nephritis with a moderate eosinophilic infiltrate without granulomatous lesion. After a few days, an open lung biopsy was also done due to respiratory deterioration. The anatomopathologic specimen demonstrated moderate fibrosis with lympho-neutrophilic infiltration and few aspecific granulomatous lesions without caseous necrosis. Sarcoïdosis was suspected and high dose oral methylprednisolone was started. Three weeks later, Mycobacterium bovis was identified by Polymerase Chain Reaction on open lung biopsy. He responded well to steroids and tuberculostatic tri-therapy. After one month of immunosuppressive treatment, renal function was resolved and hemodialysis could be discontinued. Despite the frequent use of adjuvant BCG immunotherapy, systemic complications such as hepatitis, pneumonitis, spondylodiscitis or multiorgan failure are rare $(<1 \%)$. Hematogenous dissemination which occurs a few weeks after traumatic instillations is usually suspected but not demonstrated because of absence of mycobacterium in histological specimen. Our case differs from those previously reported by the simultaneous presence of acid-fast bacilli highlighted on lung samples. We discuss the pathophysiology of BCG complications, the use of prophylactic or therapeutic treatment and recommend guidelines to prevent such complications.
\end{abstract}

Keywords: Corticosteroids; Hemodialysis; Intravesical BCG; Mycobacterium bovis; Pulmonary Granulomatosis; Renal Failure; Tubulointerstitial Nephritis; Urothelial Carcinoma

\section{Introduction}

Combined intravesical instillations of BCG remain the gold standard for intermediate and high risk non-invasive urothelial carcinoma of the bladder. Although the appearance of hematuria, dysuria or cystitis is possible, regional or systemic complications are exceptional. The hematogenous spread of mycobacterium from the bladder which occurs a few weeks after traumatic instillations is usually suspected but not demonstrated because of absence of pathogen in histological specimen. Our case correlates tubulointerstitial nephritis to mycobacterial infection by the simultaneous presence of acid-fast bacilli highlighted

${ }^{*}$ Corresponding author. on lung samples.

\section{Case Report}

In March 2012, a 76-year-old man was admitted to the hospital because of acute renal failure associated with progressive grade II dyspnea and recurrent low grade fever for 3 weeks. He also reported tiredness, an $8 \mathrm{~kg}$ weight loss and night sweats. He had a history of arterial hypertension and a non invasive (T1) transitional cell carcinoma (TCC) of the bladder. TCC was first treated during the second half of 2008 by cauterization followed by mitomycin instillations for 6 months. Because of the recurrence of polyps, the patient was treated by cauterization and one month later, he received 10 intravesical 
instillations of Baccile Calmette-Guerin (BCG) between November 2010 and July 2011. Current medications were amlodipine $10 \mathrm{mg}$ and acétylsalicylate $100 \mathrm{mg} /$ day. On admission, physical examination was unremarkable excepted fever of $37.9^{\circ} \mathrm{C}$, oliguria and few spread crackles in both lung fields and moderate edema of lower limbs. Regular hemodialysis sessions were initiated based on clinical status and blood sample evaluation.

Laboratory investigation showed hemoglobin $1.2 \mathrm{~g} / \mathrm{dL}$, a white blood cell count of $4.6 \times 10^{3} / \mu \mathrm{L}$ with moderate inflammatory signs (C-reactive protein $30 \mathrm{mg} / \mathrm{L}$, sedimentation rate $30 \mathrm{~mm} / \mathrm{h}$ ), serum creatinine $699 \mu \mathrm{mol} / \mathrm{L}$. Electrolytes and Lactatate deshydrogenase were normal, aspartate aminotransferase 51 IU/L , alcaline phosphatase $208 \mathrm{IU} / \mathrm{L}$ and gamma glutamyl transferase $269 \mathrm{IU} / \mathrm{L}$. Total protein count $47.7 \mathrm{~g} / \mathrm{L}$ with protein electrophoresis demonstrates albumin $25.1 \mathrm{~g} / \mathrm{L}$ and a thin monoclonal peak of gamma-globulin; the myelogram was normal. Serologic screenings for anti-nuclear antibody, antineutrophil cytoplasmic antibody, anti basal membrane antibody, immunoglobin A and hepatitis B or C antibodies were negative. Plasma complement and coagulation tests were also normal. Angiotensine Converting Enzyme was increased at $140 \mathrm{IU} / \mathrm{L}$ (range 8.0 - 52.0) and normal Urine analysis showed proteinuria of $0.4 \mathrm{~g} / \mathrm{L}$, sterile leucocyturia without hematuria.

The standard chest X-ray demonstrated a bilateral infiltration of the lung fields. Chest computed tomography confirmed an interstitial syndrome with a "ground glass" appearance associated with small mediastinal lymphadenopathies; there was no pleural effusion, nor micro nodular lesion. Bronchoalveolar lavage was performed and demonstrated no specific lesion; the microscopic examination revealed lympho-monocytic cells with rare neutrophils; mycobacterium culture stayed permanently sterile. A purified protein derivate (PPD) skin test was negative despite a 10 IU dose. Transesophageal cardiac echography excluded infectious endocarditis.

An abdominal CT tomography was performed and demonstrated normal kidneys, no hepatic enlargement and moderate splenomegaly. A percutaneous needle biopsy was performed and revealed tubulointerstitial nephritis with a moderate eosinophilic infiltrate without granulomatous lesion (Figure 1). Glomeruli and arterioles appeared normal; there was no histological evidence of rapidly progressive glomerulonephritis. Immunofluorescence was negative and electronic microscopy was unremarkable. After a few days, an open lung biopsy was also done due to respiratory deterioration. The anatomopathologic specimen demonstrated moderate fibrosis with lympho-neutrophilic infiltration and few aspecific granulomatous lesions without caseous necrosis or Langhans giant cells (Figure 2). Mycotic identification remained negative. Specific mycobacterium culture was started.
Sarcoïdosis was suspected and high dose oral methylprednisolone was started (1 mg/kg/day). The patient's general condition began to improve after two weeks of corticosteroid therapy in conjunction with intermittent extra-renal epuration and a supportive permanent parenteral nutrition. Fever resolved, weight increased, chest infiltrates disappeared. In the same time, the serum creatinine began to decrease as shown in Figure 3. After one month of immunosuppressive treatment, renal function resolved and hemodialysis could be discontinued. On day 27, culture of lung biopsy samples in Batec 12B medium grew acid-fast bacilli that were identified as Mycobacterium bovis by Polymerase Chain Reaction (Pasteur Institute, Brussels). Cultures of urine, alveolar fluid lavage and blood specimens remained definitely negative. Tuberculostatic agents were promptly initiated as rifadine $600 \mathrm{mg} /$ day, myambutol $1200 \mathrm{mg} / 2$ days, nicotibine $300 \mathrm{mg} /$ day and pyridoxine $250 \mathrm{mg} /$ day. Corticosteroid therapy was gradually reduced and stopped.

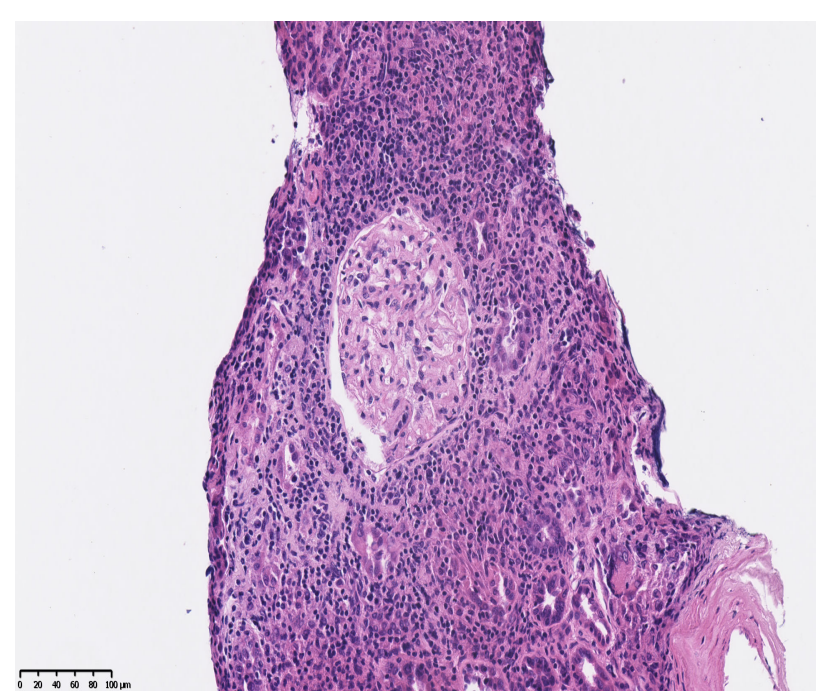

Figure 1. Kidney sample.

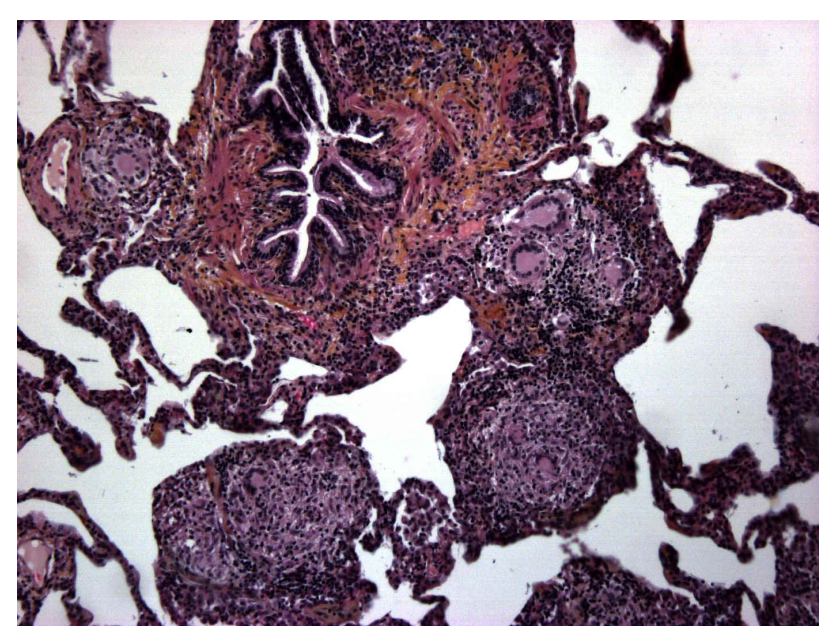

Figure 2. Lung biopsy. 


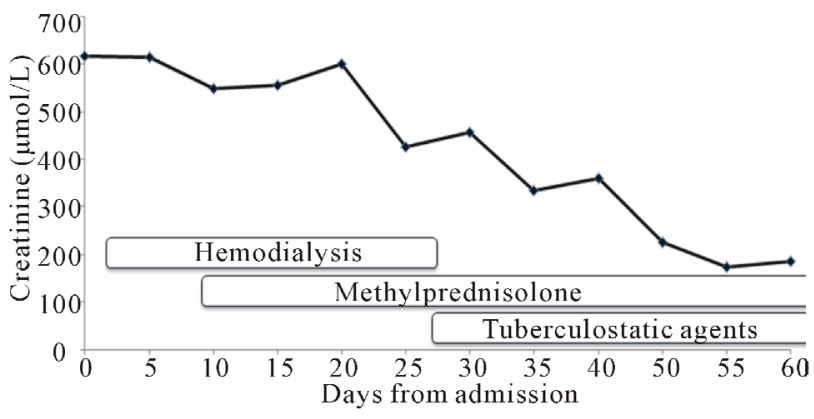

Figure 3. Recovery of renal function under corticosteroid therapy.

The patient is in good condition 6 months after initiation of antituberculosis therapy; chronic renal failure persisted with a serum creatinine of $220 \mu \mathrm{mol} / \mathrm{L}$.

\section{Discussion}

Intravesical immunotherapy with BCG has been safely used to treat recurrence of superficial transitional cell carcinoma of the bladder for more than thirty years and remains a cornerstone for the treatment of intermediate and high risk non-invasive urothelial carcinoma of the bladder [1-4]. BCG exerts its antitumor activity through induction of pro-inflammatory cytokines which may explain the flu-like syndrome [4,5] (fever, chills and arthralgia) noted in almost $20 \%$ of patients receiving therapy. Regional urological complications can occur as mycobacterium orchi-epidydimitis, prostatis or cystitis. BCG sepsis is a rare (less than 1\%, probably due to his high molecular weight) and severe complications with possible multiorgan failure are exceptional [6-9]. Ectopic localizations are also mentioned: hepatitis, pleural abscess or pneumonitis, spondylodiscitis, arthritis, mycotic aneurysms, chorioretinitis and retroperitoneal abscess. The hematogenous dissemination occurs especially after traumatic bladder instillation. Old age, acute bacterial cystitis, cumulative doses are additional risk factors which must be considered before instillations. Prophylactic drug, such as isoniazid, does not seem to reduce side effects of BCG instillations [2,3]. Contraindications include recent bladder surgery or polypectomy, recent radiotherapy treatment, hematuria, active tuberculosis, current immunosuppressive treatment and acute urine tract infection. Renal failure with histological evidence of suspected BCG toxicity is reported in twelve cases found by screening the National Library of Medecine's Medline system. In 9/12 of cases, tubule-interstitial nephritis is presented (5/12 with granuloma) and generally combined with hepatitis; mesangial glomerulonephritis, membranous glomerulonephritis and focal segmental hyalinosis are described as exceptional cases. Mycobacterial infection are usually considered as suspected responsible pathogen but not demonstrated because of ab- sence of mycobacterium in histological specimen. Our case differs from those previously reported by the simultaneous presence of acid-fast bacilli highlighted on lung samples. The evidence of Mycobacterium bovis by DNArRNA hybridization strengthens the temporal relationship between BCG instillations and kidney-lung impairment despite the unusual delayed onset complications since generally occurring a few weeks after the last instillation [5-7]. Some authors stratified into early- and late-presentation disease [10]. The first one (within 3 months) could be result from systemic infection with proliferation of the organisms causing generalized granulomatous response by repeated instillations. The second one (between 3 months and until more than 1 year) could result from reactivation of infection after successful immunologic control of early dissemination, which is rather the case of our patient. Despite severe presentation of "BCGitis", corticosteroids administration often results in a prompt recovery of both renal and lung functions. The role of corticosteroids in the treatment of the complications of BCG instillations is not clearly defined within randomized studies. Nevertheless, their use seems interesting since the objective of BCG instillation is to induce immune reaction of the host and so we can assume that symptoms may be at least partially related to this mechanism. In the present case, positive culture for Mycobacterium bovis questioned the only hypothesis of hypersensitivity reaction, reason why tuberculostatic drugs were added to corticosteroids. We also noted that, despite the absence of tuberculostatic agents during the initial phase of treatment, no proven dissemination of mycobacteria occurred.

\section{Conclusion}

Unusual complications due to adjuvant BCG immunotherapy mostly occur a few weeks after intravesical treatment. Prevention of these adverse events requires avoiding the risk factors (recent bladder surgery or polypectomy, recent radiotherapy treatment, hematuria, active tuberculosis, current immunosuppressive treatment and acute urinary tract infection) and regular control of renal function during prolonged therapy. Treatment by corticosteroids improves the outcome of patients and the question of tuberculostatic therapy should always be considered. Prophylactic drug, such as isoniazid, does not seem to prevent side effects of BCG instillations.

\section{REFERENCES}

[1] A. N. Alexandroff, A. M. Jackson, M. A. O’Donnell and K. James, "BCG Immunotherapy of Bladder Cancer: 20 Years on,” Lancet, Vol. 353, No. 9165, 1999, pp. 16891694. doi:10.1016/S0140-6736(98)07422-4

[2] M. Babjuk, W. Oosterlinck, R. Sylvester, E. Kaasinen, A. 
Böhle, J. Palou-Redorta and M. Rouprêt, "EAU Guidelines on Non-Muscle-Invasive Urothelial Carcinoma of the Bladder, the 2011 Update,” European Urology, Vol. 59, No. 6, 2011, pp. 997-1008. doi:10.1016/j.eururo.2011.03.017

[3] B. B. Houghton, V. Chalasani, D. Hayne, P. Grimison, C. S. Brown, M. I. Patel, I. D. Davis and M. R. Stockler, "Intravesical Chemotherapy Plus Bacilli Calmette-Guérin in Non-Muscle Invasive Bladder Cancer: A Systematic Review with Meta-Analysis,” BJU International, Vol. 111, No. 6, 2013, pp. 977-983. doi:10.1111/j.1464-410X.2012.11390.x

[4] T. P. Kresowik and T. S. Griffith, "Bacillus CalmetteGuerin Immunotherapy for Urothelial Carcinoma of the Bladder," Immunotherapy, Vol. 1, No. 2, 2009, pp. 281288. doi:10.2217/1750743X.1.2.281

[5] D. L. Lamm, “Complications of Bacillus Calmette-Guérin Immunotherapy," Urologic Clinics of North America, Vol. 19, No. 3, 1992, pp. 565-572

[6] M. J. Manzanera Escribano, E. Morales Ruiz, M. Odriozola Grijalba, E. Gutierrez Martínez, A. Rodriguez Antolín and M. Praga Terente, "Acute Renal Failure Due to Interstitial Nephritis after Intravesical Instillation of BCG,” Clinical and Experimental Nephrology, Vol. 11, No. 3,
2007, pp. 238-240. doi:10.1007/s10157-007-0483-6

[7] S. E. Kennedy, S. Shrikanth and J. A. Charlesworth, "Acute Granulomatous Tubulointerstitial Nephritis Caused by BCG,” Nephrology Dialysis Transplantation, Vol. 21, No. 5, 2006, pp. 1427-1429. doi:10.1093/ndt/gfk071

[8] C. G. Valentini, V. Bozzoli, A. R. Larici, L. M. Larocca, G. Delogu, G. Leone and L. Pagano, "Systemic Granulomatous Reaction Secondary to Treatment of Bladder Cancer with Bacillus Calmette-Guerin,” Mediterranean Journal of Hematology and Infectious Diseases, Vol. 4, No. 1, 2012, e2012040. doi:10.4084/mjhid.2012.040

[9] J. D. Colmerano, R. Sanjuan-Jimenez, B. Ramos and P. Morata, "Miliary Pulmonary Tuberculosis Following Intravesical BCG Therapy: Case Report and Literature Review," Diagnostic Microbiology and Infectious Disease, Vol. 74, No. 1, 2012, pp. 70-72. doi:10.1016/j.diagmicrobio.2012.05.026

[10] O. Y. Gonzalez, D. M. Musher, I. Brar, S. Furgeson, M. R. Boktour, E. J. Septimus, R. J. Hamill and E. A. Graviss, "Spectrum of Bacille Calmette-Guérin (BCG) Infection after Intravesical BCG Immunotherapy," Clinical Infectious Diseases, Vol. 36, No. 2, 2003, pp. 140-148. doi:10.1086/344908 